\title{
New aperture photometry for 217 galaxies in the Virgo and Fornax clusters $^{\star}$
}

\author{
A. Schröder ${ }^{1}$ and N. Visvanathan ${ }^{2}$ \\ 1 Astronomical Institute, University of Basel, Venusstraße 7, CH-4102 Binningen, Switzerland \\ 2 Mount Stromlo and Siding Spring Observatories, The Australian National University, Private Bag, Weston Creek \\ P.O., ACT 2611 Canberra, Australia
}

Received December 4, 1995; accepted January 30, 1996

\begin{abstract}
We present photo electric multi-aperture photometry in $U B V R I$ of 171 and 46 galaxies in the Virgo and Fornax clusters, respectively. Many of the galaxies have not been observed in at least one of these passbands before. We discuss the reduction and transformation into the Cousins photometric system as well as the extinction coefficients obtained between 1990 and 1993.
\end{abstract}

Key words: galaxies, photometry — galaxies, clusters: Virgo, Fornax

\section{Introduction}

In this paper we present a catalogue of multi-colour aperture photometry of spiral galaxies in the Virgo and Fornax clusters. In future papers total magnitudes and colours will be derived, and the data will be used to study the Tully-Fisher relation in five colours in both clusters and to obtain the relative distance of the two clusters (cf. Schröder 1995).

The aim of the observing program was to get a homogeneous set of data in different wavelengths for a complete sample of inclined spiral galaxies down to $15 \mathrm{mag}$. Though Virgo has been intensively studied, such a set of data does not exist so far (cf. de Vaucouleurs et al. 1991, hereafter RC3 ${ }^{1}$; Binggeli et al. 1985, hereafter VCC; Pierce \& Tully 1988; Yasuda et al. 1995). Fornax is less well studied, and good data are mainly found in Lauberts \& Valentijn (1989) and Mathewson et al. (1992).

We have chosen the Virgo and Fornax clusters since both are lying at about the same distance and are very close to the Local Group, so that complete lists of spiral galaxies are recorded (VCC and Ferguson 1989, hereafter FCC). The completeness is shown in their luminosity functions in Sandage et al. (1985, their Fig. 7) and Ferguson \& Sandage (1988).

Send offprint requests to: A. Schröder

${ }^{\star}$ Tables 2 and 5 are available in electronic form at the CDS via anonymous ftp 130.79.128.5

${ }^{1}$ Third Reference Catalogue of Bright Galaxies, de Vaucouleurs et al. 1991
We have obtained all data during a period of three years at Siding Spring Observatory in Australia (19901993), using the same equipment and filters at three different telescopes. We have used the Cousins photometric system (defined by the E-region stars), and the standard stars are well spread over the southern sky. The instrument and observations are described in Sect. 3.

In Sect. 4 we discuss the reduction of the data to the Cousins standard system. Though the reduction was done in a conventional way the large amount of data (more than 3000 standard star observations) makes it interesting to describe the reduction, especially the transformation into the standard system, in some detail. Moreover, in June 1991 the volcano Mt. Pinatubo in the Philippines erupted, and the raise in the extinction coefficients can be clearly seen. Finally the catalogue of the multi-aperture data is given in Sect. 5.

\section{Sample selection}

The galaxy samples have been selected from the Virgo cluster catalogue of Binggeli et al. (1985) and the Fornax cluster catalogue of Ferguson (1989). The main list of program galaxies consisted of all spiral galaxies $(\mathrm{S} 0 / \mathrm{a}-\mathrm{Sm})$ with inclinations larger than $45^{\circ}$ (calculated using $\log R$ and the equations by Heidmann et al. 1971) and with $B$-band magnitudes (as given in the catalogues) brighter than 15 mag.

To the Fornax sample we have added some possible background galaxies (from Table 3 in FCC) and six galaxies observed by Aaronson et al. (1981) as belonging to 
Table 1. Available aperture sizes in arcseconds for all three telescopes

\begin{tabular}{lrrr}
\hline diaphragm & \multicolumn{3}{c}{ aperture size [arcsec] } \\
& $2.3 \mathrm{~m}$ Tel. & 1 m Tel. & $0.6 \mathrm{~m}$ Tel. \\
\hline $8.0 \mathrm{~mm}$ & 39.2 & 89.3 & 142.7 \\
$5.6 \mathrm{~mm}$ & 27.4 & 62.5 & 99.9 \\
$4.0 \mathrm{~mm}$ & 19.6 & 44.6 & 71.4 \\
$3.5 \mathrm{~mm}$ & 17.2 & 39.1 & 62.4 \\
$3.0 \mathrm{~mm}$ & 14.7 & 33.5 & 53.5 \\
$2.5 \mathrm{~mm}$ & 12.3 & 27.9 & 44.6 \\
$2.0 \mathrm{~mm}$ & 9.8 & 22.3 & 35.7 \\
$1.5 \mathrm{~mm}$ & 7.4 & 16.7 & 26.8 \\
$1.2 \mathrm{~mm}$ & 5.9 & 13.4 & 21.4 \\
$1.0 \mathrm{~mm}$ & 4.9 & 11.2 & 17.8 \\
$0.7 \mathrm{~mm}$ & 3.4 & 7.8 & 12.5 \\
$0.5 \mathrm{~mm}$ & 2.5 & 5.6 & 8.9 \\
& & & \\
\hline
\end{tabular}

the Fornax cluster (numbered arbitrarily from A to F). A low priority list for each cluster consisted of spirals less inclined than $45^{\circ}$ (mainly between $40^{\circ}$ and $45^{\circ}$ ), galaxies with magnitudes between 15 and $16 \mathrm{mag}$, some S0 galaxies (mainly those having line widths observed in H I) and some irregular galaxies. Time permitting we observed some of these galaxies, mainly in the Fornax cluster.

\section{Instrumentation and observations}

\subsection{Instrumentation}

We have obtained the photo electric multi-aperture photometry of all galaxies at the Cassegrain foci of the $2.3 \mathrm{~m}$, $1.0 \mathrm{~m}$ and $0.6 \mathrm{~m}$ telescopes at Siding Spring Observatory. The scale of the telescopes measured at the focal plane is $4.9 \mathrm{arcsec} / \mathrm{mm}, 11.16 \mathrm{arcsec} / \mathrm{mm}$ and $17.84 \mathrm{arcsec} / \mathrm{mm}$, respectively.

We have used an automatic photo electric photometer with polarimeter, called SCAP (SCanner Aperture Photometer and Polarimeter, Visvanathan 1972, 1983). It has a rotating aperture and filter wheel controlled by a computer. A program allows the observer to set the aperture wheel at the beginning of each observation and gives a preliminary reduction at the end.

All observations were done in the single aperture mode (without a chopper). The available diaphragms and their respective sizes in arcseconds for each telescope are listed in Table 1. We have used mainly the first three diaphragms for the galaxies.

The exposure time for each filter was set within the program and was kept the same for every rotation of the filter wheel (i.e., moving the filter wheel step by step through all five filters) during the whole observing program. We have set the times so that for Sc galaxies the signal-to-noise error was about the same for all passbands for one rotation of the filter wheel. Consequently the error was higher in the $U$-band for $\mathrm{S} 0 /$ a galaxies and higher in the $I$-band for $\mathrm{Sm}$ galaxies. The ideal case is to observe the galaxy as well as the sky in all five passbands simultaneously, so that neither sky variations, seeing variations nor transparency changes would introduce error into the measurements. Hence, the total exposure time of one rotation was kept as small as possible, i.e., 4.418, 0.940, 0.752, 0.752 , and $1.128 \mathrm{~s}$ for the filters $U, B, V, R$ and $I$, respectively. Accordingly, the total integration time of a single rotation of the filter wheel was about $8 \mathrm{~s}$, and the number of rotations could be individually repeated for each object (cf. Col. (6) in Table 5).

We have used the same filters throughout the whole observing program. A new set was made by Schott for this program. The colour-glass combinations together with the use of a GaAs detector (or an S20 tube) are recommended for observing in the Cousins photometric system at the wavelengths $0.36 \mu \mathrm{m}, 0.44 \mu \mathrm{m}, 0.55 \mu \mathrm{m}, 0.64 \mu \mathrm{m}$ and $0.79 \mu \mathrm{m}$ (Bessell 1990). These combinations are as follows:
$U: 1 \mathrm{UG} 1+1 \mathrm{BG} 39$
$B: 2 \mathrm{GG} 385+1 \mathrm{BG} 12+1 \mathrm{BG} 18$
$V: 2 \mathrm{GG} 495+1 \mathrm{BG} 18+1 \mathrm{BG} 38$
$R: 2 \mathrm{OG} 570+2 \mathrm{KG} 3$
$I$ : 3RG9

Most of the observations were done using a Hamamatsu Gallium-Arsenide photomultiplier tube, together with an SSR amplifier/discriminator, except on three nights, when we had to use an S20 photomultiplier tube, because of a broken diode in the GaAs tube (15 May 17 May '93). The S20 tube is more sensitive in $B$ and $V$ compared with the GaAs tube, but less sensitive in $R$ and $I$ (Bessell 1979). Figure 1 shows the average count rate of one star, E602, observed at the same time and airmass on 15 May 93 and on 18 May 93 with the S20 and GaAs tube, respectively.

\subsection{Observations}

The data were obtained between October 1990 and June 1993. We had 182 nights, including some unassigned time, with 40,56 and 86 nights at the $2.3 \mathrm{~m}, 1.0 \mathrm{~m}$ and $0.6 \mathrm{~m}$ telescopes, respectively. Roughly $50 \%$ of the time had been useful enough to get at least some data for standard stars (which was helpful to obtain good extinction coefficients). Reasons for not observing were clouds, strong wind and instrumental problems. All galaxy observations were done under photometric conditions. The observing log is presented elsewhere (Schröder 1995).

We have used E-region standard stars (Menzies et al. 1989) together with the extension by Graham (1982) to fainter stars, and Landolt's equatorial standard stars (1983) observed in the Cousins system by Menzies et al. (1990). The E-region stars are lying at about $-45^{\circ}$ in declination and are spaced evenly by $2^{\mathrm{h}} 40^{\mathrm{m}}$ in right ascension. 


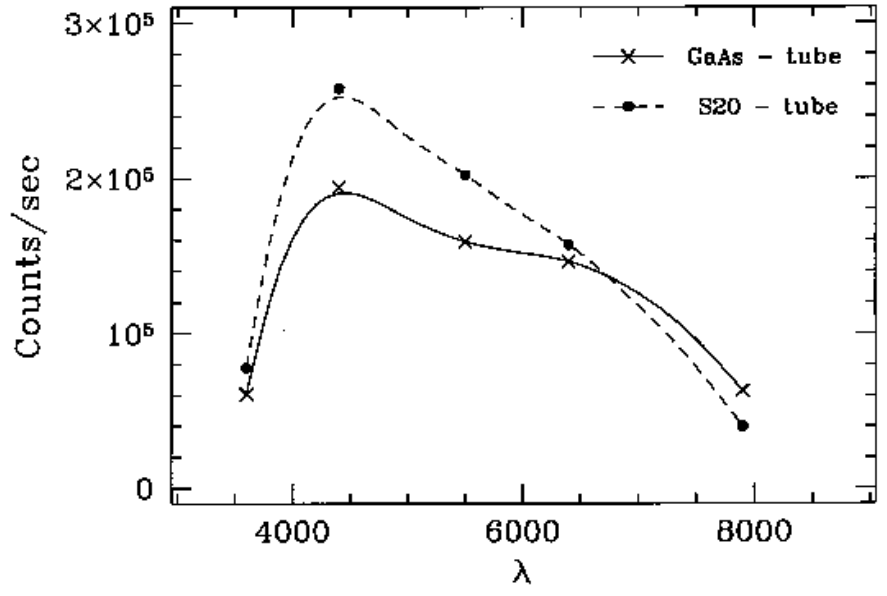

Fig. 1. Star E602 (B8 III, $V=8.1$ ), observed once with the GaAs tube (crosses) and once with the S20 tube (filled circles)

There are fewer equatorial stars and they are separated by only about $1^{\mathrm{h}}$.

All stars observed during this program are listed in Table 2 together with the standard $V$ magnitudes and $B-V$ colour (indicated by capital letters with ' $\mathrm{s}$ ' as the index). The differences between the mean values obtained from our observations and standard values are also given, as well as the number of useful observations for both the $V$ magnitude observations and the colour observations. The number of colour observations can vary by a couple of observations from one colour to the next (Schröder 1995). Stars with a prefix 'E' are E-region stars, while the others are equatorial standard stars. For star E723 the former value for $U-B$ by Menzies et al. (1980) is in better agreement with our results than the newer value, $U-B=0.544$, quoted by Menzies et al. (1989). Star HD118246 is marked as a possible variable by Landolt (1983) and Menzies et al. (1990), and has been observed only once.

On a clear night we observed between 30 and 50 standard stars. During a night a set of three or four stars near the program galaxies was monitored (in intervals of about 1.5 to 2.5 hours). We used stars in the E2-region stars when observing Fornax galaxies and equatorial standard stars for Virgo.

To accurately determine the extinction we also observed a set of standard stars in an E-region near the zenith at the beginning and at the end of a night, as well as a set of stars at high airmass, typically between 1.8 and 2.2 airmass (while the limit for galaxies was about 1.8 airmass), if the stars near the program galaxies were not sufficient. For each set stars covering a range in colour of about $0.1<B-V<1.3$ were selected. To get quickly a sufficiently low signal-to-noise error the $V$-magnitude had to be as bright as possible. However, the GaAs detector has a working limit of about 250000 to 300000 counts per second, which means an upper brightness limit for each telescope. At the $2.3 \mathrm{~m}$ telescope we used therefore a few stars from Graham (1982). The choice of equatorial stars was very limited and it was necessary to also observe fainter stars with longer exposure times.

Each object observation was bracketed by sky observations such that the total exposure time was the same for object and sky. We have chosen the exposure times so that for standard stars the signal-to-noise error was smaller than $1 \%$ and for most galaxies the signal-to-noise error was $1-2 \%$.

The instrument has a flip-flop mirror which can be moved into the light path to reflect the picture of the galaxy and its surroundings into an eyepiece or a video camera. This way the galaxies could be centered accurately (within a few arcseconds) either by eye (at the $0.6 \mathrm{~m}$ telescope) using an illuminated cross in the eyepiece, or using a cross on the TV screen.

We chose the sky region partly with the help of a print, taken from POSS films (Palomar Observatory Sky Survey) for Virgo galaxies and from ESO/ SERC films (Southern Sky Atlas) for Fornax galaxies, and partly by eye. We examined the chosen region carefully for faint stars and then kept it about the same during the observations; only at the $2.3 \mathrm{~m}$ a fixed offset could be used. However, the sky could be selected differently when a galaxy was reobserved on a different night.

We have observed 39 galaxies in Fornax and 166 in Virgo with at least three apertures. Generally around 1 to 2 hours time at the telescope was needed to observe a galaxy, depending on the type and brightness of the galaxy (this included centering of the object, observing of galaxy and sky, reading out the counts, reducing the data provisionally). If the repeatability of the observations was good and the sky variability low a time limit could be set to the observations.

In some cases we used more than three apertures (using different telescopes), mainly for big galaxies where the apertures covered only the inner part (less than $1 / 3$ in diameter), but also when there was a confusion by stars or some other problem. We used these observations together with repeated observations of bright galaxies to check for night-to-night and year-to-year variations and variations between telescopes and between the two detectors.

Only seven galaxies in Fornax and five in Virgo were observed with one or two apertures because of lack of time.

\section{Reduction}

Since we could use the same instrument with detector and filters at all three telescopes, we only expected differences in the zero point and a longterm variation of the transformation due to age effects of the detector, the filters and the mirror reflectivity. For each observing season it was necessary to use a different SSR amplifier, but no differences were found due to this in the transformation to the standard system. 
Table 2. List of standard stars (also available in electronic form at the CDS)

\begin{tabular}{|c|c|c|c|c|c|c|c|c|c|}
\hline star & $V_{\mathrm{s}}$ & $(B-V)_{\mathrm{s}}$ & $\Delta V$ & $\mathrm{n}$ & $\Delta(U-B)$ & $\Delta(B-V)$ & $\Delta(V-R)$ & $\Delta(R-I)$ & $n$ \\
\hline E101 & 7.704 & 0.087 & 0.000 & 35 & 0.006 & 0.009 & -0.004 & 0.000 & 35 \\
\hline E102 & 8.444 & 0.206 & 0.000 & 11 & -0.006 & 0.008 & 0.002 & -0.006 & 13 \\
\hline E104 & 7.455 & 0.362 & -0.004 & 15 & 0.001 & 0.004 & -0.003 & -0.008 & 15 \\
\hline E108 & 8.902 & 0.428 & -0.017 & 1 & 0.023 & -0.012 & 0.002 & -0.008 & 1 \\
\hline E109 & 8.187 & 0.518 & -0.003 & 5 & 0.017 & -0.008 & 0.011 & -0.012 & 5 \\
\hline E116 & 9.756 & 0.596 & -0.014 & 1 & 0.002 & -0.003 & 0.001 & -0.013 & 1 \\
\hline E124 & 8.928 & 1.082 & 0.005 & 6 & 0.003 & 0.002 & -0.010 & 0.008 & 7 \\
\hline $\mathrm{E} 126$ & 7.831 & 0.829 & 0.004 & 14 & 0.013 & -0.005 & 0.002 & -0.003 & 14 \\
\hline E127 & 9.347 & 0.950 & -0.005 & 8 & -0.007 & 0.004 & -0.003 & -0.004 & 8 \\
\hline $\mathrm{E} 132$ & 6.961 & 1.494 & -0.007 & 2 & 0.032 & 0.019 & -0.007 & 0.001 & 2 \\
\hline E144 & 10.886 & 0.650 & 0.002 & 24 & -0.002 & -0.013 & 0.002 & -0.009 & 24 \\
\hline E145 & 11.620 & 0.476 & -0.012 & 5 & -0.012 & 0.006 & 0.003 & -0.006 & 5 \\
\hline E $149 \mathrm{~V}$ & 11.640 & 0.564 & 0.003 & 11 & -0.003 & 0.005 & 0.002 & 0.001 & 11 \\
\hline E166 & 9.910 & 1.330 & -0.010 & 4 & -0.012 & 0.016 & -0.006 & 0.003 & 4 \\
\hline E167 & 8.323 & 1.452 & -0.003 & 15 & 0.007 & 0.019 & 0.000 & -0.006 & 17 \\
\hline E170 & 7.579 & 1.022 & 0.005 & 12 & -0.009 & -0.007 & 0.002 & 0.004 & 12 \\
\hline E1a & 12.666 & 0.615 & 0.030 & 2 & 0.008 & -0.029 & 0.011 & 0.025 & 2 \\
\hline $\mathrm{E} 205$ & 7.566 & 0.234 & 0.000 & 76 & -0.008 & 0.003 & 0.002 & -0.004 & 76 \\
\hline E207 & 8.947 & 0.340 & 0.002 & 67 & -0.007 & 0.005 & 0.003 & 0.000 & 69 \\
\hline E218 & 8.480 & 0.732 & 0.000 & 66 & 0.000 & -0.004 & 0.003 & -0.001 & 67 \\
\hline E226 & 6.934 & 0.947 & -0.003 & 1 & 0.007 & -0.007 & -0.002 & 0.012 & 1 \\
\hline $\mathrm{E} 228$ & 7.237 & 1.018 & 0.001 & 74 & -0.003 & -0.001 & -0.001 & -0.003 & 74 \\
\hline $\mathrm{E} 231$ & 7.593 & 1.305 & 0.004 & 1 & 0.023 & 0.000 & -0.013 & 0.022 & 1 \\
\hline E249 & 11.085 & 0.624 & 0.004 & 38 & 0.002 & 0.002 & -0.002 & 0.001 & 38 \\
\hline $\mathrm{E} 252$ & 7.348 & 0.179 & 0.004 & 13 & -0.011 & 0.018 & -0.006 & 0.005 & 13 \\
\hline $\mathrm{E} 253$ & 7.535 & 0.640 & -0.002 & 81 & 0.004 & -0.006 & 0.001 & 0.000 & 81 \\
\hline $\mathrm{E} 255$ & 10.312 & 0.470 & -0.001 & 33 & -0.006 & 0.002 & 0.003 & 0.004 & 33 \\
\hline E267 & 10.814 & 1.009 & 0.007 & 46 & 0.004 & -0.003 & 0.000 & -0.007 & 46 \\
\hline E271 & 8.691 & 1.075 & 0.000 & 46 & 0.001 & -0.005 & 0.007 & -0.002 & 50 \\
\hline $\mathrm{E} 272$ & 8.765 & 1.369 & 0.000 & 33 & -0.001 & 0.005 & -0.005 & 0.001 & 34 \\
\hline $\mathrm{E} 2 \mathrm{~b}$ & 11.577 & 0.529 & -0.004 & 18 & 0.012 & 0.013 & 0.003 & 0.001 & 19 \\
\hline $\mathrm{E} 21$ & 12.980 & 0.627 & 0.007 & 15 & 0.030 & 0.012 & 0.010 & -0.010 & 16 \\
\hline $\mathrm{E} 2 \mathrm{~m}$ & 13.097 & 0.806 & 0.008 & 4 & 0.010 & 0.007 & 0.000 & -0.002 & 4 \\
\hline E315 & 7.416 & 0.160 & 0.001 & 22 & 0.010 & 0.010 & -0.002 & -0.002 & 31 \\
\hline E319 & 8.986 & 0.258 & 0.005 & 38 & 0.010 & -0.002 & 0.001 & 0.001 & 38 \\
\hline E327 & 8.330 & 0.644 & 0.003 & 11 & -0.001 & 0.000 & 0.007 & -0.009 & 19 \\
\hline E329 & 8.674 & 0.626 & 0.001 & 31 & 0.004 & -0.004 & 0.002 & -0.004 & 31 \\
\hline E331 & 8.044 & 0.956 & 0.008 & 4 & -0.010 & -0.016 & 0.004 & 0.010 & 5 \\
\hline E333 & 10.060 & 1.159 & 0.007 & 24 & 0.007 & -0.001 & 0.004 & 0.001 & 25 \\
\hline $\begin{array}{l}\text { E338 } \\
\text { L }\end{array}$ & 7.978 & 1.487 & 0.006 & $\begin{array}{r}24 \\
4\end{array}$ & 0.011 & 0.004 & 0.008 & 0.001 & 5 \\
\hline E342 & 9.053 & 1.112 & -0.008 & 33 & 0.003 & 0.006 & 0.001 & 0.005 & 33 \\
\hline E346 & 7.341 & -0.111 & -0.006 & 15 & 0.001 & 0.005 & -0.001 & -0.004 & 25 \\
\hline E355 & 10.655 & 0.051 & -0.007 & 19 & -0.002 & 0.010 & -0.005 & 0.002 & 20 \\
\hline E358W & 11.570 & 0.129 & 0.004 & 3 & 0.004 & -0.009 & 0.003 & 0.013 & 3 \\
\hline E359X & 11.345 & 0.409 & $\begin{array}{r}0.0020 \\
-0.020\end{array}$ & 8 & 0.011 & 0.012 & -0.008 & 0.007 & 8 \\
\hline E383 & 10.010 & 0.305 & -0.007 & 18 & -0.010 & 0.004 & 0.001 & 0.007 & 19 \\
\hline E391 & 7.784 & 1.134 & 0.002 & 12 & 0.002 & 0.012 & 0.008 & -0.004 & 21 \\
\hline E406 & 8.348 & 0.090 & -0.004 & 20 & 0.001 & 0.006 & -0.001 & -0.003 & 33 \\
\hline E411 & 7.637 & 0.094 & 0.005 & 41 & 0.008 & 0.007 & 0.005 & -0.006 & 55 \\
\hline $\begin{array}{l}\mathrm{E} 417 \\
\end{array}$ & 9.602 & 0.200 & 0.013 & $\begin{array}{r}41 \\
9\end{array}$ & $\begin{array}{r}-0.019 \\
-0.019\end{array}$ & 0.006 & -0.005 & 0.006 & 10 \\
\hline $\mathrm{E} 425$ & 7.570 & 0.488 & 0.004 & 33 & 0.007 & -0.005 & 0.003 & 0.001 & 47 \\
\hline $\mathrm{E} 426$ & 10.003 & 0.549 & 0.001 & 21 & -0.005 & -0.010 & 0.000 & 0.005 & 26 \\
\hline $\mathrm{E} 427$ & 7.669 & 1.004 & 0.004 & 35 & -0.004 & 0.007 & 0.002 & -0.002 & 50 \\
\hline E431 & 8.506 & 1.136 & -0.003 & 20 & -0.001 & 0.003 & 0.005 & -0.006 & 38 \\
\hline $\mathrm{E} 448 \mathrm{~T}$ & 10.587 & 0.201 & 0.001 & 17 & -0.008 & 0.003 & 0.004 & -0.006 & 21 \\
\hline $\mathrm{E} 457 \mathrm{a}$ & 11.343 & 1.288 & -0.012 & 21 & 0.005 & -0.002 & -0.007 & -0.012 & 25 \\
\hline $\mathrm{E} 482$ & 8.502 & 0.910 & 0.009 & 8 & 0.004 & -0.003 & 0.006 & -0.004 & 9 \\
\hline $\mathrm{E} 484$ & 8.649 & 0.648 & 0.001 & 17 & 0.009 & -0.003 & 0.003 & -0.005 & 30 \\
\hline $\mathrm{E} 4103$ & 9.360 & 1.448 & 0.000 & 7 & 0.025 & 0.016 & -0.008 & 0.010 & 9 \\
\hline $\mathrm{E} 4106$ & $\begin{array}{r}11.097 \\
\end{array}$ & 0.300 & -0.016 & 6 & -0.016 & 0.006 & -0.011 & 0.012 & 6 \\
\hline E4d & 12.595 & 0.638 & -0.011 & 5 & 0.011 & 0.008 & -0.012 & -0.013 & 5 \\
\hline E504 & 8.557 & 0.147 & 0.025 & 3 & -0.011 & 0.004 & 0.006 & -0.004 & 3 \\
\hline E508 & 10.000 & 0.366 & 0.007 & 3 & -0.026 & -0.009 & -0.001 & -0.011 & 5 \\
\hline E516 & 7.850 & 0.542 & 0.008 & 16 & 0.008 & -0.007 & 0.005 & 0.000 & 17 \\
\hline E520 & 7.528 & 0.560 & 0.010 & 5 & 0.016 & -0.007 & 0.003 & 0.009 & 7 \\
\hline E522 & 10.086 & 0.376 & 0.000 & 5 & -0.022 & -0.001 & 0.002 & 0.012 & 6 \\
\hline E528 & 7.550 & 1.108 & 0.002 & 19 & 0.002 & 0.003 & 0.003 & -0.006 & 23 \\
\hline E529 & 8.347 & 1.027 & 0.016 & 3 & 0.000 & -0.013 & 0.000 & 0.002 & 3 \\
\hline E535 & 8.623 & 1.430 & 0.008 & 3 & -0.006 & 0.003 & -0.004 & -0.010 & 3 \\
\hline E541 & 7.312 & 0.012 & -0.002 & 21 & -0.002 & 0.003 & 0.000 & 0.003 & 24 \\
\hline E545 & 10.614 & 0.161 & -0.011 & 4 & -0.026 & 0.012 & 0.001 & 0.000 & 5 \\
\hline $\begin{array}{l}\mathrm{E} 547 \\
\end{array}$ & 10.824 & 0.651 & -0.003 & $\begin{array}{r}4 \\
10\end{array}$ & -0.003 & -0.004 & 0.001 & $\begin{array}{r}0.0010 \\
-0.010\end{array}$ & 13 \\
\hline
\end{tabular}

A coincidence correction (or dead-time correction) has been applied to all observations. It becomes important (that is, greater than 1\%) at count rates of about 250000 counts per second, so it is preferable that observations do not exceed such rates. The correction of $K=610^{-8}$ is applied to the observations as

$$
C_{\text {corr }}=\frac{C_{\text {obs }}}{1-K \cdot C_{\mathrm{obs}}}
$$

where $C$ is the count rate per second.

The equations for extinction correction and transformation, which are the best for this observing site, filters and detector (Bessell, priv. comm.), are of the form (with $X$ as airmass):

Extinction correction:

$$
\begin{aligned}
v_{\mathrm{o}} & =v-k_{v}^{\prime} X \\
(b-v)_{\mathrm{o}} & =(b-v)-k_{b v}^{\prime} X-k_{b v}^{\prime \prime} X(B-V)_{\mathrm{o}} \\
(u-b)_{\mathrm{o}} & =(u-b)-k_{u b}^{\prime} X \\
(v-r)_{\mathrm{o}} & =(v-r)-k_{v r}^{\prime} X \\
(r-i)_{\mathrm{o}} & =(r-i)-k_{r i}^{\prime} X
\end{aligned}
$$


Table 2. continued

\begin{tabular}{|c|c|c|c|c|c|c|c|c|c|}
\hline star & $V_{\mathrm{S}}$ & $(B-V)_{\mathrm{S}}$ & $\Delta V$ & $\mathrm{n}$ & $\Delta(U-B)$ & $\Delta(B-V)$ & $\Delta(V-R)$ & $\Delta(R-I)$ & $n$ \\
\hline E $548 \mathrm{U}$ & 10.519 & 1.260 & 0.008 & 2 & 0.024 & 0.015 & -0.001 & -0.007 & 2 \\
\hline E556 & 10.882 & 0.990 & -0.012 & 9 & 0.006 & 0.001 & -0.003 & 0.005 & 12 \\
\hline E572 & 8.940 & 0.670 & 0.017 & 3 & 0.012 & -0.004 & 0.000 & -0.002 & 3 \\
\hline E584 & 7.431 & 1.280 & 0.002 & 12 & -0.006 & 0.015 & 0.000 & -0.005 & 13 \\
\hline $\mathrm{E} 602$ & 8.089 & -0.026 & 0.007 & 4 & -0.007 & 0.002 & -0.001 & 0.000 & 4 \\
\hline E607 & 8.780 & 0.119 & 0.002 & 28 & -0.009 & -0.003 & 0.003 & 0.004 & 30 \\
\hline E611 & 10.102 & 0.066 & 0.002 & 14 & -0.027 & 0.003 & 0.000 & 0.005 & 17 \\
\hline E612 & 7.888 & 0.159 & -0.002 & 29 & 0.006 & 0.004 & -0.004 & 0.003 & 30 \\
\hline $\mathrm{E} 622$ & 8.118 & 0.568 & 0.006 & 28 & 0.000 & -0.010 & 0.001 & -0.002 & 28 \\
\hline E623 & 8.996 & 0.534 & 0.008 & 3 & -0.005 & -0.005 & -0.004 & 0.002 & 5 \\
\hline $\mathrm{E} 627$ & 7.314 & 0.973 & -0.002 & 26 & -0.008 & 0.000 & -0.010 & 0.012 & 27 \\
\hline E628 & 7.522 & 0.939 & -0.002 & 14 & -0.001 & 0.006 & -0.005 & 0.008 & 15 \\
\hline E634 & 10.154 & 0.815 & 0.002 & 13 & 0.008 & -0.008 & 0.004 & -0.001 & 16 \\
\hline E651 & 7.355 & -0.018 & -0.002 & 10 & -0.001 & -0.009 & -0.002 & 0.013 & 11 \\
\hline $\mathrm{E} 652$ & 7.206 & 0.540 & -0.003 & 23 & 0.003 & -0.005 & -0.002 & 0.008 & 24 \\
\hline $\mathrm{E} 653$ & 7.089 & 0.580 & -0.001 & 6 & -0.029 & 0.007 & -0.009 & 0.013 & 6 \\
\hline $\mathrm{E} 661$ & 10.150 & 1.238 & 0.020 & 8 & 0.022 & 0.014 & -0.002 & 0.011 & 10 \\
\hline E675 & 10.002 & 1.175 & -0.001 & 12 & 0.022 & 0.003 & -0.015 & 0.017 & 15 \\
\hline E697 & 8.361 & 1.198 & 0.007 & 29 & 0.005 & 0.008 & -0.001 & 0.005 & 31 \\
\hline E705 & 8.493 & 0.108 & -0.007 & 9 & 0.025 & -0.012 & -0.001 & -0.007 & 14 \\
\hline E708 & 10.548 & 0.169 & 0.004 & 11 & -0.011 & 0.006 & -0.001 & -0.001 & 14 \\
\hline E708W & 10.548 & 0.169 & 0.032 & 2 & -0.010 & -0.015 & -0.003 & -0.012 & 2 \\
\hline E723 & 7.213 & 0.624 & 0.011 & 6 & 0.024 & 0.003 & 0.004 & 0.003 & 6 \\
\hline E728 & 8.153 & 0.698 & -0.002 & 15 & -0.007 & -0.008 & 0.002 & 0.007 & 29 \\
\hline E732 & 7.645 & 1.248 & -0.001 & 7 & 0.007 & 0.010 & -0.003 & 0.006 & 15 \\
\hline E734 & 8.130 & 1.144 & -0.009 & 15 & -0.003 & 0.001 & -0.001 & 0.007 & 29 \\
\hline E746 & 7.252 & 0.088 & -0.004 & 7 & 0.008 & 0.004 & -0.004 & 0.014 & 17 \\
\hline E750 & 7.549 & -0.056 & -0.009 & 2 & 0.009 & -0.004 & 0.003 & 0.010 & 10 \\
\hline E751 & 8.344 & 0.130 & 0.000 & 1 & -0.001 & 0.001 & 0.002 & 0.010 & 3 \\
\hline $\mathrm{E} 752 \mathrm{X}$ & 10.777 & 0.024 & -0.011 & 12 & 0.068 & 0.003 & 0.008 & 0.002 & 15 \\
\hline E756 & 10.406 & 1.125 & -0.005 & 10 & -0.015 & 0.008 & -0.016 & 0.014 & 12 \\
\hline E772 & 10.442 & 0.624 & -0.009 & 9 & 0.004 & -0.002 & -0.007 & 0.009 & 12 \\
\hline E793 & $\begin{array}{r}9.542 \\
\end{array}$ & 1.286 & -0.008 & 1 & -0.008 & 0.010 & -0.008 & 0.025 & 3 \\
\hline $\mathrm{E} 7 \mathrm{~b}$ & 10.956 & 0.612 & -0.003 & 5 & -0.040 & 0.010 & -0.005 & 0.002 & 5 \\
\hline $\mathrm{E} 7 \mathrm{~m}$ & 12.498 & 1.316 & 0.017 & 6 & 0.015 & 0.010 & -0.001 & 0.010 & 6 \\
\hline E 847 & 10.620 & 0.518 & 0.011 & 1 & -0.032 & -0.001 & -0.009 & 0.020 & 1 \\
\hline E848 & 10.707 & 0.914 & 0.014 & 1 & -0.010 & 0.004 & -0.009 & 0.006 & 1 \\
\hline E901 & 8.056 & 0.047 & 0.003 & 2 & 0.009 & 0.013 & 0.014 & -0.013 & 2 \\
\hline E902 & 10.875 & -0.043 & -0.009 & 1 & 0.028 & 0.000 & 0.000 & -0.008 & 1 \\
\hline E904 & 8.928 & 0.340 & -0.007 & 2 & 0.015 & 0.007 & 0.002 & -0.005 & 2 \\
\hline E905 & 7.717 & 0.362 & 0.002 & 3 & 0.010 & 0.001 & -0.004 & 0.003 & 5 \\
\hline E906 & 8.891 & 0.302 & 0.000 & 3 & 0.011 & 0.002 & 0.000 & -0.006 & 3 \\
\hline E907 & 7.530 & 0.374 & 0.000 & 3 & 0.001 & -0.003 & 0.000 & 0.000 & 3 \\
\hline E917 & 7.221 & 0.601 & 0.003 & 2 & 0.010 & -0.004 & 0.001 & 0.001 & 2 \\
\hline E922 & 10.345 & 0.516 & -0.006 & 1 & -0.012 & -0.012 & -0.012 & 0.025 & 1 \\
\hline E925 & 7.064 & 0.989 & -0.003 & 3 & 0.003 & 0.006 & -0.005 & 0.000 & 3 \\
\hline E926 & 9.230 & 1.010 & 0.004 & 1 & 0.005 & -0.002 & -0.003 & 0.004 & 1 \\
\hline E928 & 10.372 & 1.060 & -0.011 & 2 & -0.045 & -0.015 & -0.016 & -0.002 & 2 \\
\hline E930 & 6.849 & 1.116 & -0.002 & 3 & 0.004 & 0.004 & -0.002 & -0.006 & 3 \\
\hline E931 & 7.249 & 1.448 & -0.002 & 5 & -0.018 & 0.017 & -0.009 & -0.002 & 5 \\
\hline E933 & 8.866 & 1.093 & 0.003 & 1 & 0.011 & 0.011 & 0.002 & -0.013 & 1 \\
\hline E939 & 8.704 & 1.554 & 0.014 & 1 & 0.007 & 0.025 & 0.000 & -0.006 & 1 \\
\hline E941 & 7.233 & 0.460 & -0.002 & 3 & -0.007 & -0.001 & 0.002 & 0.002 & 3 \\
\hline E945 & 7.258 & 0.158 & -0.005 & 5 & 0.000 & 0.009 & -0.004 & 0.001 & 5 \\
\hline E946 & 7.292 & 0.141 & -0.001 & 2 & 0.009 & 0.005 & 0.003 & -0.011 & 2 \\
\hline E947U & 10.685 & 0.591 & -0.002 & 2 & 0.015 & -0.003 & 0.005 & -0.023 & 2 \\
\hline E956 & 8.341 & 1.077 & 0.002 & 1 & 0.001 & -0.003 & 0.000 & -0.006 & 1 \\
\hline E969 & 7.770 & 0.744 & 0.001 & 3 & -0.002 & 0.008 & -0.004 & 0.000 & 3 \\
\hline E973 & 6.910 & 0.068 & 0.015 & 2 & -0.003 & -0.001 & 0.010 & -0.003 & 2 \\
\hline E977 & 7.680 & 0.429 & 0.023 & 1 & 0.015 & -0.017 & 0.026 & -0.018 & 1 \\
\hline 103462 & 10.114 & 0.569 & 0.002 & 84 & -0.014 & 0.001 & 0.001 & 0.002 & 86 \\
\hline 103483 & 8.353 & 0.425 & -0.002 & 282 & -0.009 & 0.000 & -0.001 & 0.000 & 286 \\
\hline 104337 & 11.211 & 0.784 & -0.005 & 107 & -0.003 & -0.004 & -0.001 & -0.004 & 113 \\
\hline 104461 & 9.706 & 0.484 & 0.002 & 406 & 0.007 & -0.003 & 0.000 & 0.001 & 416 \\
\hline 104598 & 11.480 & 1.123 & 0.005 & 18 & -0.111 & -0.002 & 0.000 & 0.005 & 19 \\
\hline 10528 & 8.354 & 1.050 & -0.003 & 199 & -0.004 & -0.001 & -0.001 & -0.001 & 199 \\
\hline 105448 & 9.174 & 0.248 & -0.004 & 4 & -0.008 & -0.001 & -0.014 & 0.012 & 5 \\
\hline 105663 & 8.766 & 0.346 & 0.000 & 68 & -0.011 & -0.006 & -0.004 & 0.000 & 68 \\
\hline 105815 & 11.450 & 0.393 & 0.001 & 80 & 0.018 & -0.004 & 0.004 & -0.007 & 86 \\
\hline BD 52529 & 9.585 & 1.262 & -0.010 & 14 & 0.002 & $\begin{array}{l}-0.003 \\
-0.003\end{array}$ & -0.002 & 0.001 & 16 \\
\hline HD118246 & 8.054 & -0.149 & -0.014 & 1 & -0.048 & -0.004 & -0.012 & 0.002 & 1 \\
\hline \multicolumn{10}{|c|}{ stars observed with the $\mathrm{S} 20$ tube: } \\
\hline 103483 & 8.353 & 0.425 & 0.027 & 11 & -0.033 & -0.005 & 0.000 & 0.021 & 11 \\
\hline 104461 & 9.706 & 0.484 & -0.033 & 17 & 0.031 & 0.007 & -0.003 & -0.024 & 17 \\
\hline 10528 & 8.354 & 1.050 & 0.014 & 17 & -0.006 & -0.003 & 0.007 & 0.011 & 17 \\
\hline 105663 & 8.766 & 0.346 & 0.005 & 8 & -0.005 & -0.004 & -0.008 & -0.006 & 8 \\
\hline
\end{tabular}

Transformation:

$$
\begin{aligned}
V_{\mathrm{o}} & =A_{0}+v_{\mathrm{o}}+B_{0}(b-v)_{\mathrm{o}} \\
(U-B)_{\mathrm{o}} & =A_{1}+(u-b)_{\mathrm{o}}+B_{1}(b-v)_{\mathrm{o}} \\
(B-V)_{\mathrm{o}} & =A_{2}+B_{2}(b-v)_{\mathrm{o}} \\
(V-R)_{\mathrm{o}} & =A_{3}+B_{3}(v-r)_{\mathrm{o}} \\
(R-I)_{\mathrm{o}} & =A_{4}+B_{4}(r-i)_{\mathrm{o}}
\end{aligned}
$$

In this notation small letters stand for the data in the natural system, that is, data without any correction except an approximate zero point, which is the same for all telescopes and nights; an index 'o' denotes extinction corrected values, and capital letters were used for data transformed into the standard system.

Different reduction procedures are described in the literature (e.g., Hardie 1962; Harris et al. 1981). We have used a combination of these, reducing the data in an iterative way and using all the stars from one night together.

We always worked with the residuals with respect to the standard system. We excluded 'bad' stars with large deviations, for example, stars affected by clouds or bad 
seeing, or stars with uncertain standard values. The nights of an observing season were divided into runs. A run is a row of successive nights, often at different telescopes, and separated from the next run by the nights around full moon. It consisted typically of 4-20 nights. If a run had only one useful night we added it to the following or the previous run.

First we determined the extinction coefficients using all stars of one night. We corrected the data for extinction with a median value that was taken of all nights in a run. Then we determined a zero point for each night separately and used all stars of one observing season to derive the transformation coefficients. In the second iteration we applied the transformation correction to the uncorrected data and determined again the extinction coefficient. The following transformation corrections showed little difference to the first set, so a third iteration gave no further improvement.

\subsection{Extinction}

We decided to use all stars of one night to determine the extinction correction, contrary to other methods where each star with measurements at different airmass is considered separately (see Hardie 1962). Our method is less time consuming since we already got a good extinction coefficient after half an hour of observing time. The scatter in the data due to other corrections is largely removed in the iterative process.

The resulting extinction coefficients showed that for photometric nights the repeatability was good. No change in extinction coefficients with position in the sky could be found. We have therefore taken the median extinction coefficient of a run and only excluded nights where the stars covered a small range in airmass $\left(X_{\max } \lesssim 1.4\right)$. This way an extinction correction for every night could be obtained, and errors caused by large deviations avoided. We preferred the mean value if only a couple of good nights of one run were available.

A second order extinction coefficient, depending on colour, had to be determined for $(b-v)$. We derived this coefficient using a linear regression after applying the first order extinction correction. Again we determined the median value for each run.

No differences in the extinction coefficients between the nights using the S20 tube and the following nights, observed again with the GaAs tube, could be found, as expected, so we could take the median values for the whole run as usual.

The night-to-night and year-to-year variations of the extinction coefficients in the $V$-band and the colours are shown in Fig. 2, where the extinction coefficients are plotted versus Julian Date. The observations began on 14 Oct. 1990 (J.D. $=2448178$ ), and the last observing day was 22 Jun. 1993 (J.D. = 2449160 ). The errors are those derived from the linear regression. Nights with insufficient data (i.e., not used for the median value) are not shown. The median extinction coefficient for each run is listed in Table 3 together with the median over all good nights of each of the three observing seasons. For comparison the average site values for Siding Spring Observatory are also given (Bessell 1990, priv. comm.).

The increase in the extinction coefficient in $\mathrm{V}$ between 1991 and 1992 is clearly caused by the eruption of Mt. Pinatubo in the Philippines during June 1991 (for details see Bernard et al. 1991). In 1993 the extinction was still higher than in 1991, though comparable with the site value. Grothues \& Gochermann (1992) found similar results in their observations made at La Silla in Chile between 1990 and 1992. The increase in extinction is almost wavelength independent, though we found a slight increase towards longer wavelengths. Comparing the first and second observing season gives $\Delta k_{u}=0.06 \mathrm{mag} / \mathrm{AM}, \Delta k_{b}=$ $0.08 \mathrm{mag} / \mathrm{AM}, \Delta k_{v}=0.08 \mathrm{mag} / \mathrm{AM}, \Delta k_{r}=0.09 \mathrm{mag} / \mathrm{AM}$, and $\Delta k_{i}=0.10 \mathrm{mag} / \mathrm{AM}$ (where AM denotes the airmass). A comparison of the first and third observing season still shows a mean increase in extinction of about $0.05 \mathrm{mag} / \mathrm{AM}: 0.04,0.05,0.05,0.06$ and $0.07 \mathrm{mag} / \mathrm{AM}$ in $U, B, V, R$ and $I$, respectively.

This shows the importance of measuring individual extinction coefficients, even two years after such an eruption. Also, the differences of the first, unaffected, observing season to the site values show the advantage of measured extinction coefficients. Beside the rather big difference in $k_{v}^{\prime}$, the second extinction coefficient for $B-V, k_{b v}^{\prime \prime}$, is clearly positive throughout our observations, though very small, while the site value is negative. There are also occasional strong variations in the extinction, for instance in Oct. '90 and in Dec. '91 (at J.D. $\sim 2448180$ and $\sim 2448590$ ), that have to be taken into account (cf. Burki et al. 1995).

As an example, the difference in using the median value for the 1st observing season and the site value in $V$ would be $\Delta V=-0.05 \mathrm{mag}$ for an airmass of 1.0 (the site value gives brighter magnitudes) and $\Delta V=-0.08 \mathrm{mag}$ for an airmass of 1.8 ; for the colour $B-V$ the difference would be $\Delta(B-V)=-0.05 \mathrm{mag}$ for an airmass of 1.0 and $\Delta(B-V)=-0.09 \mathrm{mag}$ for an airmass of 1.8 .

\subsection{Transformation}

To derive the transformation corrections we plotted the residuals of all for extinction and zero point corrected stars from one observing season, regardless of the telescope used, as a function of standard values. We found no differences in the distribution of the residuals when we plotted them for each telescope separately.

With roughly 1000 data points per observing season the transformation to the standard system is well determined. Figure 3 shows the transformation for each observing season for the GaAs and the S20 tube. Galaxy measurements are in the range of $0.05<B-V<1.20$, 
Table 3. Median extinction coefficients for each run and observing season and the site values. The second number in each column is the error in units of $10^{-3} \mathrm{mag}$

\begin{tabular}{lrrrrrrrrrrrr}
\hline \multicolumn{1}{c}{ Run } & \multicolumn{2}{c}{$k_{v}^{\prime}$} & \multicolumn{2}{c}{$k_{u-b}^{\prime}$} & \multicolumn{2}{c}{$k_{b-v}^{\prime}$} & $k_{b-v}^{\prime \prime}$ & & $k_{v-r}^{\prime}$ & & $k_{r-i}^{\prime}$ \\
\hline Oct '90 & 0.218 & 17 & 0.274 & 3 & 0.109 & 3 & 0.002 & 2 & 0.049 & 3 & 0.037 & 9 \\
Nov '90 & 0.141 & 5 & 0.236 & 7 & 0.107 & 4 & 0.019 & 5 & 0.041 & 8 & 0.036 & 1 \\
Dec '90 & 0.127 & 6 & 0.260 & 5 & 0.104 & 9 & 0.011 & 3 & 0.046 & 7 & 0.057 & 5 \\
Feb '91 & 0.142 & 12 & 0.258 & 4 & 0.156 & 10 & 0.006 & 5 & 0.046 & 5 & 0.041 & 4 \\
Mar '91 & 0.126 & 9 & 0.258 & 6 & 0.117 & 5 & 0.013 & 1 & 0.041 & 5 & 0.040 & 5 \\
Apr '91 & 0.129 & 6 & 0.243 & 3 & 0.127 & 6 & 0.006 & 3 & 0.040 & 4 & 0.048 & 4 \\
1. obs. season & 0.135 & 6 & 0.257 & 3 & 0.118 & 4 & 0.009 & 1 & 0.045 & 2 & 0.041 & 2 \\
Dec/Jan '91/'92 & 0.277 & 27 & 0.250 & 6 & 0.121 & 5 & 0.001 & 3 & 0.042 & 4 & 0.050 & 6 \\
Feb/Mar '92 & 0.210 & 4 & 0.233 & 3 & 0.119 & 3 & 0.004 & 1 & 0.041 & 3 & 0.036 & 3 \\
Mar/Apr '92 & 0.196 & 6 & 0.241 & 4 & 0.116 & 2 & 0.002 & 2 & 0.036 & 3 & 0.030 & 0 \\
Apr/May '92 & 0.228 & 5 & 0.240 & 7 & 0.114 & 3 & 0.005 & 4 & 0.039 & 4 & 0.043 & 4 \\
May/Jun '92 & 0.224 & 9 & 0.245 & 2 & 0.115 & - & 0.003 & 6 & 0.044 & 13 & 0.044 & 9 \\
2. obs. season & 0.218 & 4 & 0.238 & 3 & 0.117 & 2 & 0.004 & 1 & 0.039 & 2 & 0.035 & 2 \\
& & & & & & & & & & & \\
Feb '93 & 0.187 & 3 & 0.237 & 4 & 0.114 & 4 & 0.003 & 2 & 0.034 & 3 & 0.033 & 3 \\
Mar '93 & 0.197 & 2 & 0.252 & 6 & 0.116 & 6 & 0.005 & 3 & 0.039 & 3 & 0.035 & 4 \\
Apr '93 & 0.182 & 5 & 0.258 & 2 & 0.110 & 3 & 0.010 & 1 & 0.045 & 3 & 0.031 & 2 \\
May '93 & 0.184 & 4 & 0.247 & 4 & 0.113 & 5 & 0.001 & 1 & 0.042 & 1 & 0.028 & 5 \\
Jun '93 & 0.189 & 16 & 0.237 & 10 & 0.114 & 17 & 0.010 & 5 & 0.036 & 2 & 0.041 & 7 \\
3. obs. season & 0.187 & 2 & 0.248 & 2 & 0.113 & 2 & 0.006 & 1 & 0.039 & 1 & 0.032 & 1 \\
site values & 0.18 & & 0.28 & & 0.13 & & -0.03 & & 0.030 & & 0.035 \\
\hline
\end{tabular}

$-0.05<V-R<0.85$ and $-0.10<R-I<0.90 ;$ the limits are indicated as dashed lines in the plots.

Since we only observed about 20 different standard stars on the three nights with the S20 tube, the transformation for this tube is less accurate. Furthermore, the zero point shifted considerably when the telescope was moved over the sky. However, this shift only became obvious when the telescope was moved a large amount, so within one standard star region and within each cluster the shift was negligible. This was also the case when following the equatorial standard stars near Virgo slowly during the night (see also Fig. 4). Nevertheless, this caused a problem when determining the transformation, since we had to use a different zero point for each E-region separately.

While a linear regression was sufficient for the transformation of $v_{\mathrm{o}},(b-v)_{\mathrm{o}},(v-r)_{\mathrm{o}}$ and $(r-i)_{\mathrm{o}}$ to the standard system it was necessary to use a 5 th order polynomial for the transformation of $(u-b)_{\mathrm{o}}$. A 3rd order polynomial would not fit the slope for $(B-V)_{\mathrm{s}}<0$ correctly, which is well determined in the third observing season. The few data points obtained with the S20 tube are sufficiently fitted with a 3rd order polynomial.

We excluded the following stars for the $U-B$ reduction, since their standard values $(U-B)_{\mathrm{s}}$ are considerably different to our observations: star 104598 (large error in standard value), E752X (very blue and rather faint, in a crowded region) and E928 (faint, observed with $0.6 \mathrm{~m}$ telescope).
The transformation coefficients for each observing run are shown in Table 4 , where the coefficients $B_{0}$ to $B_{4}$ are explained at the beginning of this section. $C_{1}$ to $F_{1}$ are coefficients of terms in $B-V$ of higher orders. The changes from one observing season to the next are only small, but there is a clear tendency. This can be an effect of the decreasing efficiency of the GaAs tube (10-15\%), which had been noticed during the observing time, combined with other effects like degrading transmission of the $U$ filter and changing reflectivity of the mirrors. Aside from this long term effect the system was very stable.

The transformation coefficients for the S20 tube were distinctly different and larger than the ones used for the GaAs tube.

\subsection{Zero point}

The residuals of the stars of each night, after correcting for extinction and transformation, were plotted versus time to check for a change in zero point. Often a slight temporal variation was noticeable in the data, especially at those telescopes with polar mounting $(0.6 \mathrm{~m}$ and $1.0 \mathrm{~m})$. This could have been caused by large and fast movements of the telescope (e.g., from the zenith to an airmass of 2.0) and with it by a movement of the detector (which means that the melting dry ice inside shifted) and by a change in temperature (see Menzies 1986).

The change in zero point was often small enough $(\leq 0.1 \mathrm{mag})$ and could be easily corrected as time 


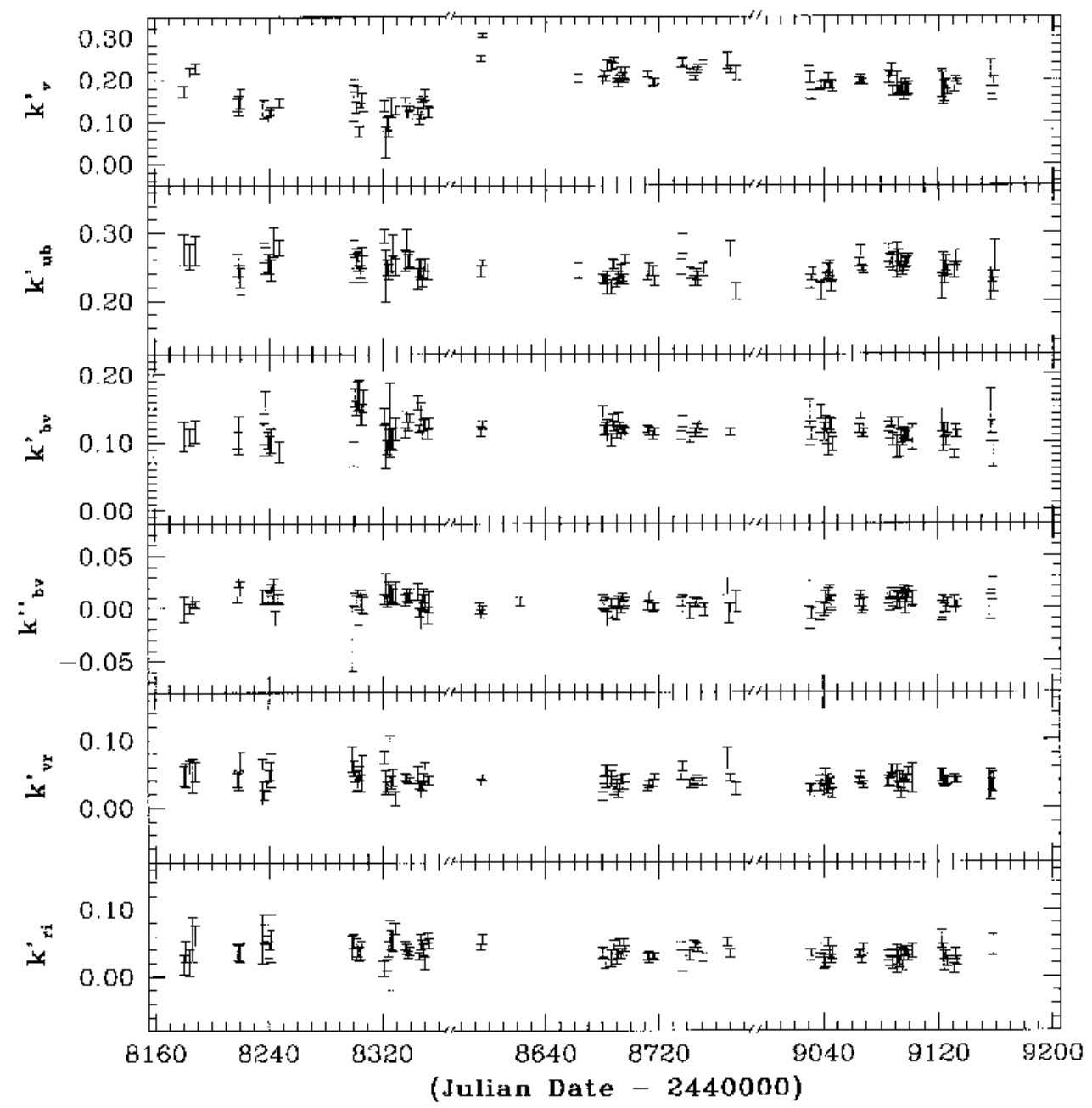

Fig. 2. Extinction coefficients of all three observing seasons

dependent since the standard stars were lying close to the galaxies and since there were no large telescope movements between the objects to be observed. Also, no clear dependence on any other parameter, such as position in the sky, could be found, except for three successive nights at the $1 \mathrm{~m}$ telescope, where a slight correction for azimuth was necessary. (It has to be noted that since the objects were at nearly the same position - in R.A. and dec - a dependence on time also implies a dependence on the location in the sky - altitude and azimuth - and vice versa). The three or four equatorial standard stars, which covered almost two hours in right ascension, showed no bigger scatter in their observations at a certain time than stars of one E-region.

In Fig. 4 the residuals of three different nights are plotted versus local time. E-region stars and equatorial stars are indicated by open circles and crosses, respectively. The first example is the night of 24 Mar. 91 at the $2.3 \mathrm{~m}$ telescope. The scatter for each group of stars is very small and no time correction was needed. The second example is the night of 14 Mar. 92 at the $1 \mathrm{~m}$ telescope. The correction for Fornax (E-region stars) and Virgo (equatorial stars) is shown. Successive nights at the same telescope can show similar patterns; in addition, there are variations of these two examples, some of them with a slightly non-linear time dependence.

The third example shows the night of 17 May 93 at the $1 \mathrm{~m}$ telescope when we used the S20 tube. The scatter for each group of observations is larger than when using the GaAs tube, and there is a considerable change in zero point between the E-region and the equatorial stars, as well as between the five stars of the E6-region and those of the E7-region (E4 and E5 had been observed in the beginning of the night, and E6 and E7 in the end).

The colours showed no or only little variation in the zero point. 
1. obs. season

2. obs. season

3. obs. season
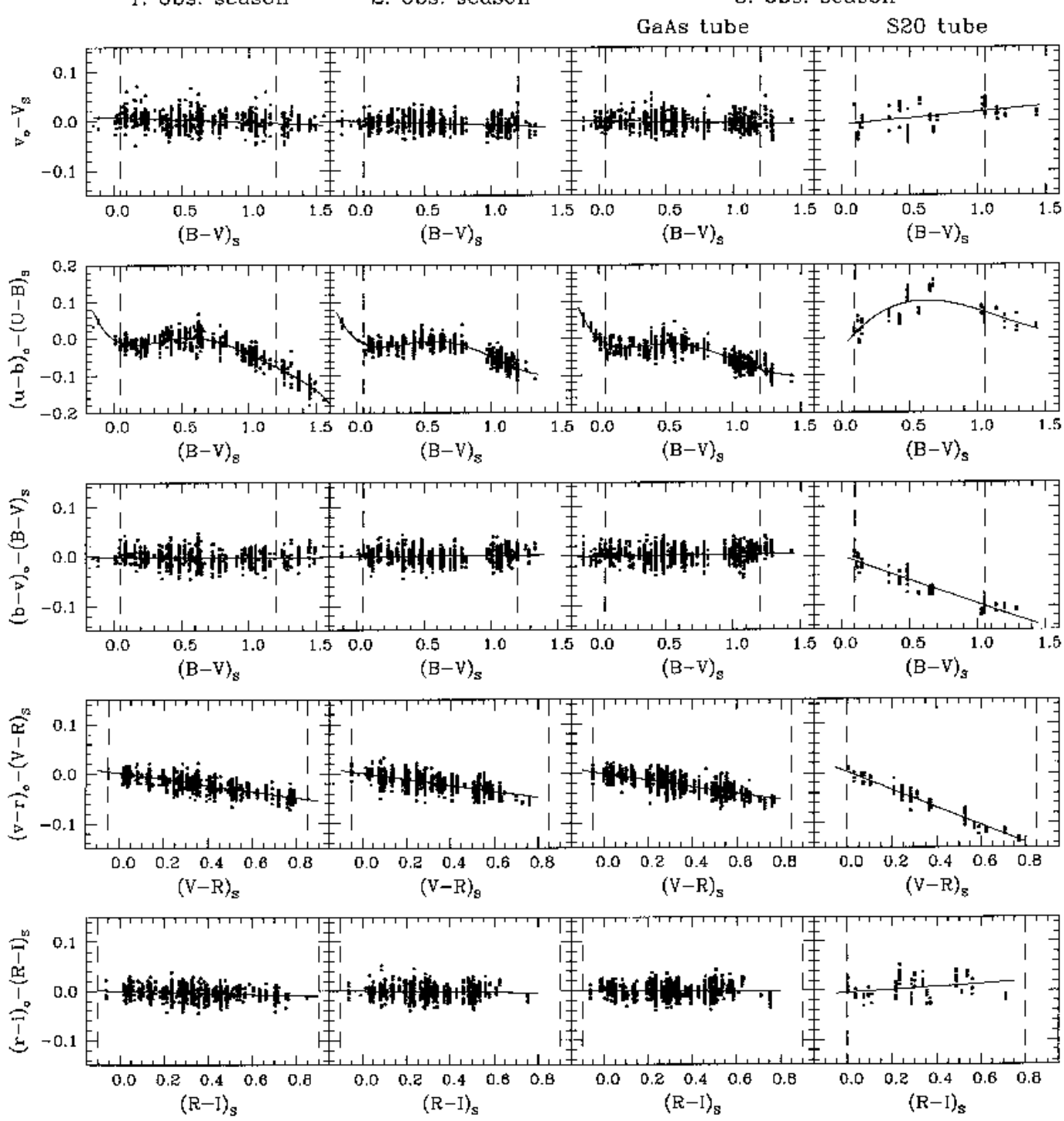

Fig. 3. Transformation of the standard stars for all three observing seasons and for the S20 tube separately. Galaxy measurements are lying in the range indicated by the dashed lines

\section{Results}

The scatter of the final residuals in the $V$ magnitudes and all colours are between $0.011 \mathrm{mag}$ and $0.016 \mathrm{mag}$. A remaining dependence of the residuals on airmass and standard values is negligible for the program galaxies.

Table 5 (available in electronic form) lists the aperture photometry of all observed galaxies. The columns are as follows:

Columns (1) and (2): The number of the galaxy in the respective catalogue (VCC and FCC) and one alternative name of the galaxy: NGC, IC, ESO, UGC or other identifications, in this order of preference.

Columns (3) and (4): Right ascension and declination (epoch 1950.0).
Column (5): The morphological type of the galaxy from VCC or FCC. For those galaxies that are in neither of the catalogues the types from RC3 were used.

Column (6): Aperture size in units of arcseconds.

Column (7): The logarithm of the aperture in units of 0.1 arcminute.

Column (8): The total integration time in units of rotations of the filter wheel: one rotation corresponds to 4.418 , $0.940,0.752,0.752$ and $1.128 \mathrm{~s}$ for the $U, B, V, R$ and $I$ filter, respectively.

Columns (9)-(13): The B-magnitudes and colours. A colon indicates a signal-to-noise error of $0.10 \mathrm{mag}$ and more; brackets are used for data that has been excluded from further data reduction for various reasons, such as a) too low exposure times, b) exceptionally high sky counts 
Table 4. The transformation coefficients for each observing season and for the last season separately for the GaAs tube and the S20 tube

\begin{tabular}{|c|c|c|c|c|c|c|c|c|c|}
\hline Observing season & $\begin{array}{c}V \\
B_{0}\end{array}$ & $B_{1}$ & $C_{1}$ & $\begin{array}{c}U-B \\
D_{1}\end{array}$ & $E_{1}$ & $F_{1}$ & $\begin{array}{c}B-V \\
B_{2}\end{array}$ & $\begin{array}{c}V-R \\
B_{3}\end{array}$ & $\begin{array}{c}R-I \\
B_{4}\end{array}$ \\
\hline 1. obs. season ('90/'91) & 0.010 & 0.269 & -1.312 & 2.115 & -1.337 & 0.306 & 1.003 & 1.058 & 1.011 \\
\hline $\begin{array}{l}\text { 2. obs. season ('91/'92) } \\
\text { 3. obs. season ('93): }\end{array}$ & 0.008 & 0.281 & -1.149 & 1.597 & -0.805 & 0.124 & 0.998 & 1.058 & 1.005 \\
\hline $\begin{array}{l}\text { GaAs - tube } \\
\text { S20 - tube }\end{array}$ & $\begin{array}{r}0.004 \\
-0.026\end{array}$ & $\begin{array}{r}0.329 \\
-0.491\end{array}$ & $\begin{array}{r}-1.370 \\
0.537\end{array}$ & $\begin{array}{r}2.118 \\
-0.155\end{array}$ & -1.295 & 0.277 & $\begin{array}{l}0.996 \\
1.095\end{array}$ & $\begin{array}{l}1.066 \\
1.178\end{array}$ & $\begin{array}{l}1.000 \\
0.971\end{array}$ \\
\hline
\end{tabular}

(i.e., a large aperture), c) a bright star in the aperture was not measured or light from a star scattered into or out of the aperture, d) the aperture was not centered accurately, e) error too high. Sometimes only one or two passbands were affected. All data in brackets should be rejected.

Column (14): A note indicates if a star (or more than one star) was included in and subtracted from the aperture, and if the aperture was observed with the S20 tube. Brackets around S20 indicate an aperture that was partly observed with the S20 and with the GaAs tube. Two

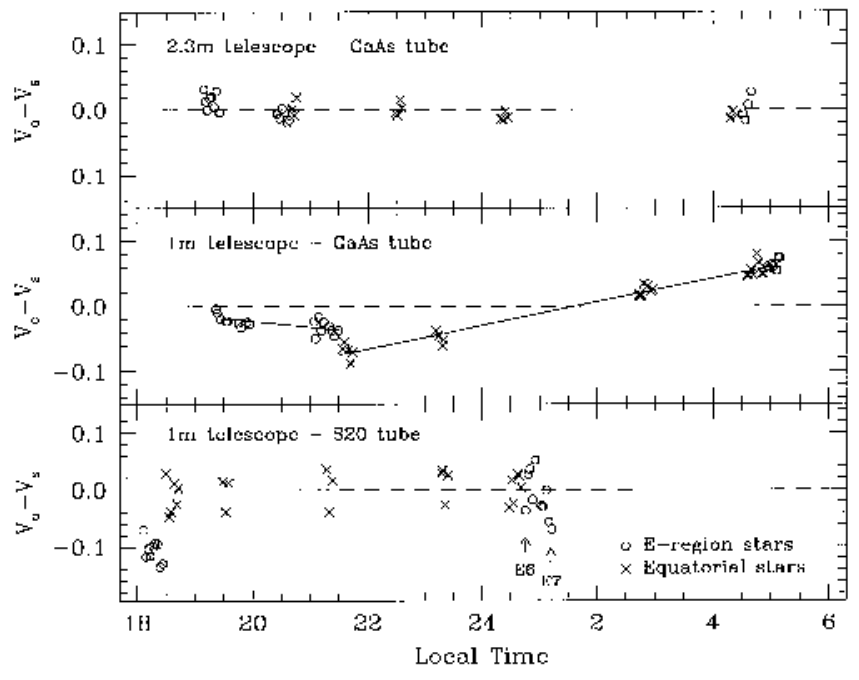

Fig. 4. Zero point variations in $V$ for a typical night at the $2.3 \mathrm{~m}$ telescope on 24 Mar. 91 (upper panel), and at the $1 \mathrm{~m}$ telescope with the GaAs tube on 14 Mar. 92 (middle panel). The lower panel shows the same for one of the three nights at the $1 \mathrm{~m}$ telescope with the S20 tube (17 May 93)

galaxies, which were observed with both the S20 and the GaAs tube, do not show any difference in the observations. Also, the three nights are in good agreement with each other, and the resulting data agree well with the literature.

The errors of the magnitudes are calculated from the signal-to-noise ratio. They are usually smaller than
$0.04 \mathrm{mag}$ with bright observations having smaller errors than fainter ones, as expected (cf. Schröder 1995).

The data of 104 galaxies are in very good agreement within the errors with data from the catalogue by Longo \& de Vaucouleurs (1983). A more detailed comparison with data in the literature will be shown in the following paper (Schröder 1996) where the total magnitudes and colours will be derived.

Judging from the FCC the observed sample of spiral galaxies in Fornax is complete for inclinations larger than $40^{\circ}$ and $m_{B}<15.5 \mathrm{mag}$, including S0 galaxies with observed line widths $(n=34)$. In addition there are 12 galaxies with smaller inclinations, fainter magnitudes, or being lenticular or irregular galaxies.

In Virgo the observed sample of spiral galaxies is complete for inclinations larger than $45^{\circ}$ and $m_{B}<14.0 \mathrm{mag}$ $(n=90)$ except for one interacting galaxy pair (V_1673 and V_1676), which is difficult to separate in photometry as well as line width measurement. Of 55 spiral galaxies in the range $14.0 \mathrm{mag}<m_{B}<15.0 \mathrm{mag}$ three late type spirals are missing, with the brightest of it having no line width measurement. Furthermore nine late type spirals and one Sa (without line width measurement) with $m_{B}>15.0$ have not been observed.

All S0 galaxies with line width measurements have been observed except for one bright galaxy $\left(V_{-} 801\right)$ and three galaxies fainter than $m_{B}=14.8(n=13)$. Additionally seven galaxies that are amorphous or have inclinations smaller than $45^{\circ}$ and one spiral galaxy that is not in the VCC (NGC 4651, numbered as V_A in Table 5) have been observed.

\section{Summary}

We give a catalogue of multi-aperture data in the five passbands $U B V R I$ for 171 and 46 galaxies in the Virgo and Fornax clusters, respectively, obtained between 1990 and 1993. For the reduction of the data we obtained more than 3000 standard star observations. The resulting extinction coefficients show a raise of about $0.08 \mathrm{mag} / \mathrm{AM}$ between the first half year of 1991 and 1992 caused by the eruption 
of the Pinatubo volcano, and they are still higher of about $0.05 \mathrm{mag} / \mathrm{AM}$ in the first half year of 1993. The transformation to the Cousins standard system is very stable during the three years of observing and is well determined.

Acknowledgements. Our thanks to the MSSSO time allocation committee for assigning large numbers of nights for this project. We also thank the staff at SSO for all their help during the long observing runs. A. Schröder thanks Mount Stromlo and Siding Spring Observatory for their hospitality between 1990 and 1993, G.A. Tammann for theencouragement throughout the project, and the Swiss National Science Foundation for financial support.

\section{References}

Aaronson M., Dawe J.A., Dickens R.J., Mould J.R., Murray J.B., 1981, MNRAS 195, 1

Bernard A., Demaiffe D., Mattielli N., Punongbayan R.S., 1991, Nat 354, 139

Bessell M.S., 1979, PASP 91, 589

Bessell M.S., 1990, PASP 102, 1181

Binggeli B., Sandage A., Tammann G.A., 1985, AJ 90, 1681 (VCC)

Burki G., Rufener F., Burnet M., et al., 1995, The ESO Messenger 80, 34

Ferguson H.C., 1989, AJ 98, 367 (FCC)

Ferguson H.C., Sandage A., 1988, AJ 96, 1520

Graham J.A., 1982, PASP 94, 244

Grothues H.-G., Gochermann J., 1992, The ESO Messenger 68, 43
Hardie, R.H., 1962, In: Hiltner, W.A. (ed.) Astronomical Techniques. University of Chicago Press, Chicago, p. 178

Harris W.E., Fitzgerald M.P., Reed B.C., 1981, PASP 93, 507

Heidmann J., Heidmann N., de Vaucouleurs G., 1971, Mem. R. Astron. Soc. 75, 85

Landolt A.U., 1983, AJ 88, 439

Lauberts A., Valentijn E.A., 1989, The Surface Photometry Catalogue of the ESO-Uppsala Galaxies, ESO, Garching $(\mathrm{LV})$

Longo G., de Vaucouleurs A., 1983, The University of Texas Monographs in Astronomy No. 3

Mathewson D.S., Ford V.L., Buchhorn M., 1992, ApJS 81, 413 (MFB)

Menzies J.W., 1986, In: Hearnshaw J.B., Cottrell P.L. (eds.), Proc. IAU Symp. 118, Instrumentation and Research Programmes for Small Telescopes. Reidel, Dordrecht, p. 57

Menzies J.W., Banfield R.M., Laing J.D., 1980, SAAO 5, 149

Menzies J.W., Cousins A.W.J., Banfield R.M., Laing J.D., 1989, SAAO 13, 1

Menzies J.W., Marang F., Laing J.D., Coulson I.M., Engelbrecht C.A., 1990, MNRAS 248, 642

Pierce M.J., Tully R.B., 1988, ApJ 330, 579

Sandage A., Binggeli B., Tammann G.A., 1985, AJ 90, 1759

Schröder A., 1995, PhD thesis, University of Basel

Schröder A., 1996 (in preparation)

de Vaucouleurs G., de Vaucouleurs A., Corwin H.G., Buta R.J., Paturel G., Fouqué P., 1991, Third Reference Catalogue of Bright Galaxies. Springer, New York (RC3)

Visvanathan N., 1972, PASP 84, 248

Visvanathan N., 1983, ApJ 275, 430 